\title{
The Levitation Mass Method - A precision force measurement method using an optical interferometer and a levitated mass
}

\author{
Yusaku Fujii ${ }^{\text {a,* }}$ \\ ${ }^{a}$ School of Science and Technology, Gunma University, 1-5-1 Tenjin-cho, Kiryu, Gunma 376-8515, Japan \\ *Corresponding Author: fujii@el.gunma-u.ac.jp
}

\begin{abstract}
A method for precision force measurement, i.e. the levitation mass method (LMM), which has been proposed and improved by authors, are reviewed. An rigid object is levitated with a small friction using an aero-static linear bearing. The total force acting on the object (the moving part of the bearing) is measured as the product of mass and acceleration of the object, i.e. F=Ma. In the method, only the Doppler shift frequency of the laser beam reflected by the object is accurately measured using an optical interferometer. Subsequently, the velocity, position, acceleration, and inertial force of the object are calculated from this frequency.
\end{abstract}

